\title{
Rapid method of recording transmission spectra of pumped active media samples
}

\author{
A.V. Lovchev, A.K. Naumov, R.D. Aglyamov and E. Yu. Tselischeva \\ Kazan Federal University, Department of Physics, 420008 Kazan, Russia
}

\begin{abstract}
The application of the phenomenon of a laser-induced spark for spectroscopy is described. The characteristics of such a light source, its advantages are given. Examples of using the source in real experiments on measuring absorption from the excited state of various materials are given.
\end{abstract}

To analyze the optical properties of materials, the pump-probe method is useful. This method uses relatively weak probe radiation when relatively powerful pumping radiation is exposed to the sample. For example, the properties of a potential active element in lasing mode, while the probe radiation wavelength is the same as assumed lasing wavelength with real pumping.

However, if the probe radiation is continuous or quasi-continuous, then for a pulsed pump it becomes problematic to detect a change in the material spectral properties associated with the pumping of a signal against continuous probe radiation. Or problem associated with both pulsed sources synchronization. There is also a problem with the mutual influence of pump and probe beams. Due to the fact that the radiation must pass through one zone, the problem of their independent registration arises, which is especially important at a low difference between probe and pump wavelengths.

When implementing the pump-probe method, it is not always possible to find a pulsed laser source of the probe radiation and synchronize it with a pump source. Therefore, in the case of laser pumping of samples in an experiment, it is convenient to use a laser-induced spark [1] as probe radiation. It is relatively easy to arrange such a source close to the sample in an experiment, a branched pump beam can be used as a spark inducing source, the intensity of the spark is close repeating the intensity of the pumping radiation, as seen in Fig. 1, measured with oscilloscope Tektronix DPO 7354 (band 3,5GHz, 40GS/s) and sensors AlphaLas UPD-50-UD. Nd:YAG laser pulse and spark were used for the both channels measurements. Cables length and distances between sensors and objects of measurements were equals at this case.

The spectrum of such a source is wide enough for a wide variety of problems, and the dimensions of the laser spark, shown at photo, allow focus it with good efficiency. It is also possible to excite a spark in a transparent cuvette filled with a specific gas composition at a pressure different from atmospheric pressure, which allows both to increase the brightness of such a light source and to expand its spectrum.

Using the experimental setup spectra of $\mathrm{Ce}: \mathrm{LiY}_{\mathrm{x}} \mathrm{Lu}_{(1-\mathrm{x})} \mathrm{F}_{4}$ crystal differential transmission at various pumping energy densities have been measured, that not been studied before. The results of this measurement are presented in figure 2. From these spectra it is clear that absorption in wavelength range $245-285 \mathrm{~nm}$ is observed due to 
excited state absorption process (wavelength range 3 ). It is really so because this band completely disappear when the pumping light is turned off and also the intensity of the maximum of this band depends nonlinearly on pumping energy density.

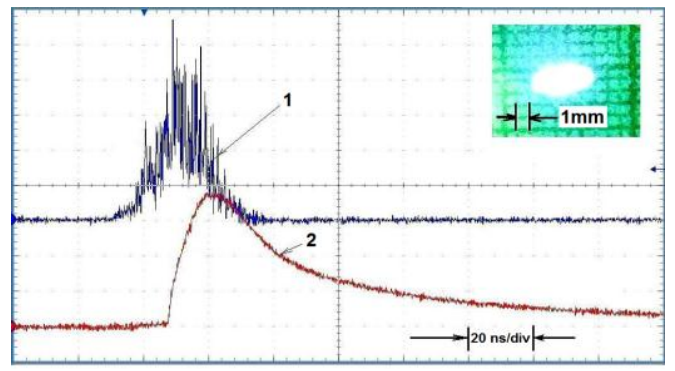

Fig. 1. Intensity of the laser pulse and induced from them spark in time domain, and the photo of the laser induced spark.

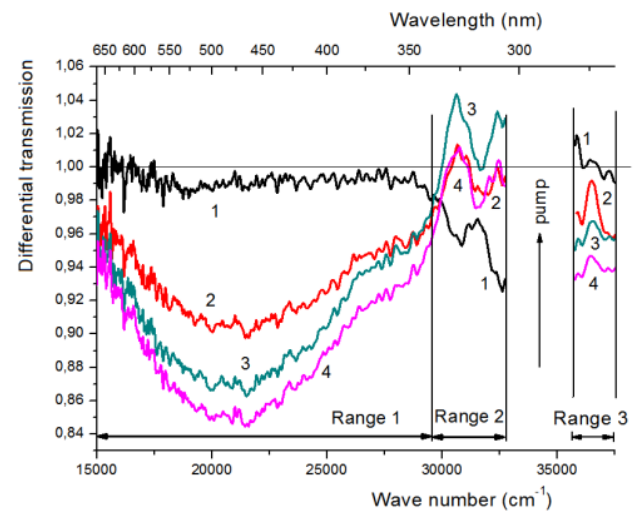

Fig. 2. The spectra of differential transmission of the $\mathrm{Ce}: \mathrm{LiY}_{\mathrm{x}} \mathrm{Lu}_{(1-\mathrm{x})} \mathrm{F}_{4}$ crystal on excitation energy density: $1-0,2 \mathrm{~J} / \mathrm{cm}^{2} ; 2-0,8 \mathrm{~J} / \mathrm{cm}^{2} ; 3-0,9 \mathrm{~J} / \mathrm{cm}^{2} ; 4-1,0 \mathrm{~J} / \mathrm{cm}^{2}$.

In the wavelength range $300-330 \mathrm{~nm}$ differential transmission of the sample is larger than unity (wavelength range 2). It can be concluded that the observed spectrum is the spectrum of gain since this range coincides with the luminescence band of $\mathrm{Ce}^{3+}$ in this crystal, and because it also disappears when the excitation light is switched off. In addition, its intensity depends linearly on the excitation density with some saturation at high intensities. In the wavelength range 1 from 320 to $700 \mathrm{~nm}$ the wide absorption bands were observed. These absorption bands also disappear when excitation light is switched off. We associate this band with absorption of short-living color centers.

Also, this method was tested in a number of other experiments, namely when measuring the gain of active media activated by rare-earth ions [2-3] and measuring the absorption from the excited state at various pump sources in different spectral regions.

\section{References}

1. Tran X. Phuoc, Optics and Lasers in Engineering. 43, 113 (2005)

2. V.G. Gorieva, S.L. Korableva and V.V. Semashko, Optical Materials Express, 6, 1146 (2016)

3. R.D. Aglyamov at al. Russian Patent RU2654390 (2017) 